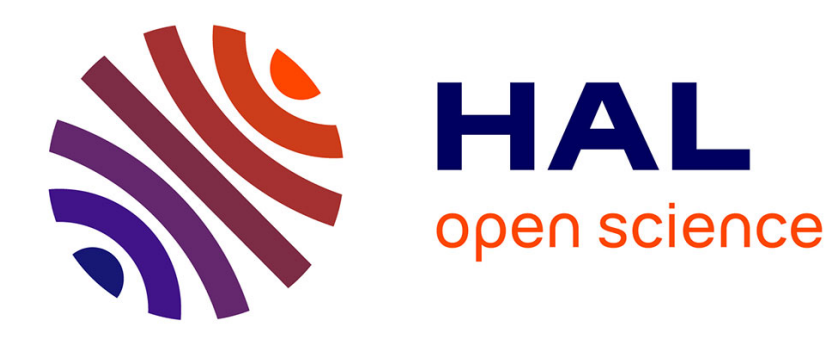

\title{
Magnetic multilayers : fundamental and practical aspects
}

\author{
R. Krishnan
}

\section{To cite this version:}

R. Krishnan. Magnetic multilayers: fundamental and practical aspects. Journal de Physique IV Proceedings, 1992, 02 (C3), pp.C3-159-C3-167. 10.1051/jp4:1992323 . jpa-00251528

\section{HAL Id: jpa-00251528 https://hal.science/jpa-00251528}

Submitted on 1 Jan 1992

HAL is a multi-disciplinary open access archive for the deposit and dissemination of scientific research documents, whether they are published or not. The documents may come from teaching and research institutions in France or abroad, or from public or private research centers.
L'archive ouverte pluridisciplinaire HAL, est destinée au dépôt et à la diffusion de documents scientifiques de niveau recherche, publiés ou non, émanant des établissements d'enseignement et de recherche français ou étrangers, des laboratoires publics ou privés. 


\title{
Magnetic multilayers: fundamental and practical aspects
}

\author{
R. KRISHNAN
}

Laboratoire de Magnétisme et Matériaux Magnétiques, C.N.R.S., 92195 Meudon, France

\begin{abstract}
After a brief introduction we describe the preparation of the multilayers by evaporation under ultra high vacuum conditions and their characterisation. We then discuss the magnetic and magneto-optical properties of some multilayer systems such as, $\mathrm{Ni} / \mathrm{Ag}, \mathrm{Fe} / \mathrm{Ag}, \mathrm{Co} / \mathrm{Ag}$ and $\mathrm{Co} / \mathrm{Pt}$. The last one is quite interesting and is a potential candidate for magneto-optical information storage, particularly capable of working in the shorter wave length of light. We describe particularly the various anisotropies such as the bulk and surface ones and the magnetostriction as well.
\end{abstract}

\section{INTRODUCTION}

Magnetic metallic superlattices, multilayered or compositionally modulated films are of great interest both from the fundamental and technological points of view ${ }^{1}$. Research on these new materials started early in the past decade but has quickly gained interest from several laboratories all over the world. The nano-structured materials raise a certain number of questions as to redefining the properties in a quasi atomic scale. This poses some problems because any property of an atom arises from the nature and the symmetry of the neighbouring environment and to have a precise knowledge of this is vital to predict the properties. Thanks to sophisticated characterization techniques and atomic probes, such as, intense synchrotron sources, pulsed neutron sources for magnetic study, polarized electron spectroscopy,scanning tunnelling microscopy, field emission microscopy etc., which are fast developing, one can hopefully look forward to the future to gain a better understanding of these aspects. There are several interesting aspects which arise from the fact that the magnetic layers are only a few atomic planes thick and the architectural nature of the multilayers. For example, it has been shown theoretically that the magnetic moment in mono-layers of some transition metals could show some enhancement and some experimental results have been published, not only on superlattices but also on sputtered multilayers $2-4$. Another interesting and important aspect is the appearance of strong surface anisotropy which is also of great technological interest. The notion of surface anisotropy was first pointed out by Néel 5 as early as 1954 and experimental values have been reported by Gradmann 6 . This interfacial anisotropy arises from the surface atoms with broken symmetry and which begin to dominate the bulk anisotropy when the layer thickness approaches a few atomic layers 7 . Such anisotropy is found not only in the superlattices 8 but also in the compositionally modulated layers 9,10 . It is well known that the anisotropy and the magnetostriction both arise from the spin orbit coupling. The microscopic mechanisms of the magnetostriction however are far more complicated than that encountered in the magnetic anistropy and treating such a problem is still an open question for the theorists 11 . One normally expects magnetostricton when anisotropy is present. Can one then expect the surface magnetostriction a logical analogue of the surface anisotropy? This problem has been addressed by us before 12 . Recently attention has been drawn also to the fact that the interlayer coupling between the adjacent magnetic layers could be either ferromagnetic or antiferromagnetic depending on the electronic structure and the thickness of the spacer layer. In such cases one could also expect large magnetoresistance which is of great interest from technological point of view 13-14. This topic is also of current interest and is being actively pursued in many laboratories. 
We describe in this paper the results of our experimental work on some chosen multilayer systems such as $\mathrm{Ni} / \mathrm{Ag}, \mathrm{Fe} / \mathrm{Ag}, \mathrm{Co} / \mathrm{Ag}, \mathrm{Co} / \mathrm{Pt}$ and $\mathrm{Ni} / \mathrm{Pt}$. The system based on $\mathrm{Ag}$ is interesting because $\mathrm{Ag}$ does not alloy with the transition metals. Therefore the alloying at the interface could be avoided. The Co/Pt sysiem is chosen because of its potential application for magnetoptical storage medium. We will briefly describe the preparation techniques and the methods of characterization. In discussing the properties we would try to bring out the complexities of the problems concerned with the anisotropy and the magnetostriction in these materials.

\section{EXPERIMENTAL DETAILS}

The multilayers were prepared by using dual e-guns fitted in an ultra high vacuum chamber 15 where the starting pressure is $1-2 \times 10^{-10}$ torr and which during film deposition could be maintained below $5.10^{-9}$ torr. Various materials such as, glass, silicon and $\mathrm{MgO}(100)$ were used as substrates and maintained at temperatures in the range 300 to $500 \mathrm{~K}$. The rate of evaporation of about $0.05 \mathrm{~nm}$ and the final thicknesses were controlled by using calibrated quartz oscillators. In the range of thickness where bulk densities are obtained, the estimation of the film thickness is accurate to about $\pm 3 \%$. The multilayers were enclosed between the $\mathrm{Ag}$ and Pt layers. The transition metal layer thickness was in the range 5 to $50 \AA$. The thicknesses of $\mathrm{Ag}$ layer is $50 \AA$ and that of $\mathrm{Pt}$ is varied in the range 10 to $16 \AA$. The number of bi-layers was varied from 5 to 30 .

Low and high angle X-ray diffraction was carried out to check the quality of the samples. Planar and cross sectional transmission electron microscopy were performed on selected samples.

The magnetization (M) and the $\mathrm{M}-\mathrm{H}$ loops were measured with a vibration sample magnetometer and the anisotropy with a torque meter in the temperature range 5 to $290 \mathrm{~K}$.

In some cases we also studied the polar Kerr and Faraday loops also with a He-Ne laser using a magneto-optic set up capable of measuring a rotation of $10^{-4} \mathrm{deg}$.

The magnetostriction was measured using the strain modulated ferromagnetic resonance (SFMR) method 16 where the strain periodic in time is applied to the sample placed in a microwave cavity. This causes the modulation of the resonance absorption line position. As a consequence of the magnetoelastic coupling, the intensity of the signal obtained after phase sensitive detection is proportional to the strain modulation depth $\mathrm{m}_{\mathrm{S}}$. The magnetically modulated FMR signal $\mathrm{m}_{0}$ is also simultaneously recorded. The magnetostriction constants can be obtained by comparing the intensity of the SFMR $\left(\mathrm{I}_{\mathrm{S}}\right)$ and FMR $\left(\mathrm{I}_{0}\right)$ signals.

\section{Magnetization}

\section{RESULTS AND DISCUSSIONS}

In the case of $\mathrm{Ni} / \mathrm{Ag}$, it is seen that the magnetization in the multilayers (ML) is relatively high even for thin Ni layers as compared to published results on systems such as, Ni/Mo etc.. This shows that the interface purity is also relatively high. However in the case of Co one does observe an increase in the magnetization for Co layers close to $20 \AA 10$.

\section{Anisotropy and Magnetostriction}

In the case of ML, the thickness ( $t$ ) dependence of the anisotropy can be written, after the phenomenological model as follows:

$$
\mathrm{K}^{\mathrm{eff}}=\mathrm{K}_{\mathrm{v}}+2 \mathrm{~K}_{\mathrm{S}} \mathrm{t} \text {, }
$$

where, $\mathrm{K}^{\text {eff }}$ is the measured anisotropy, $\mathrm{K}_{\mathrm{V}}$ is the volume anisotropy and is expressed as $\mathrm{K}_{\mathrm{V}}=$ $K_{\text {int }}+2 \pi M^{2}$ and $K_{\text {int }}$ contains the crystalline and magnetoelastic anisotropies. $K_{s}$ is the surface 
anisotropy which is counted twice for the two interfaces as this arises from the surface atoms which see a broken symmetry.

Fig. 1 shows the variation of $\mathrm{K}_{\mathrm{eff}} \times \mathrm{t}(\mathrm{Ni})$ as a function of $\mathrm{t}(\mathrm{Ni})$. The straight line verifies the model. However it is seen that the extrapolation shows that $\mathrm{K}_{\mathrm{s}}$ is zero. It can be hence concluded that the surface $\mathrm{Ni}$ atoms do not contribute to the anisotropy in this system.

The analysis of the results on the anisotropy for Fe/ $\mathrm{Ag} 18$ yields a positive contribution to $\mathrm{K}_{\mathrm{S}}$ of $+0.16 \mathrm{erg} . \mathrm{cm}^{-2}$, as shown by the linear extrapolation in Fig. 2 .

For Co/Ag samples 18 when $\mathrm{K}_{\text {eff }} \times \mathrm{t}$ vs $\mathrm{t}(\mathrm{Co})$ is plotted, a linear behaviour is observed as shown in Fig. 3. The analysis of this data gives $K_{s}=+0.16 \mathrm{erg} . \mathrm{cm}^{2}$ which is slightly smaller than 0.25 erg. $\mathrm{cm}^{2}$ reported recently for $\mathrm{Co} / \mathrm{Ag}$ multilayers ${ }^{19}$. From the slope of the straight line we get $\mathrm{K}_{\mathrm{v}}=-1 \times 10^{7} \mathrm{erg} . \mathrm{cm}^{-3}$ would give for our samples (where $2 \pi \mathrm{M}^{2}=1.25 \times 10^{7} \mathrm{erg} \cdot \mathrm{cm}^{-3}$ ) $\mathrm{K}_{\mathrm{int}}=0.25 \times 10^{7} \mathrm{erg} \cdot \mathrm{cm}^{-3}$. This also agrees well with value calculated from the experimental torque curve.

Now let us discuss the magnetostriction. Fig. 4, shows that $\lambda_{\mathrm{s}}$ decreases linearly with $\mathrm{t}(\mathrm{Ni})^{-1}$ and it can be expressed, as in the case of the anisotropy, as a sum of contributions from the bulk magnetostriction and the surface magnetostriction 20 . Hence we can write,

$$
\lambda_{s}=\lambda_{s}(\text { bulk })+2 \lambda_{s}(\text { surf }) / t
$$

Our experimental results can be described by the following relation,

$$
\lambda_{\mathrm{s}}=-35.6 \times 10^{-6}+35.4 \times 10^{-5}(\AA) . \mathrm{t}(\mathrm{Ni})^{-1}
$$

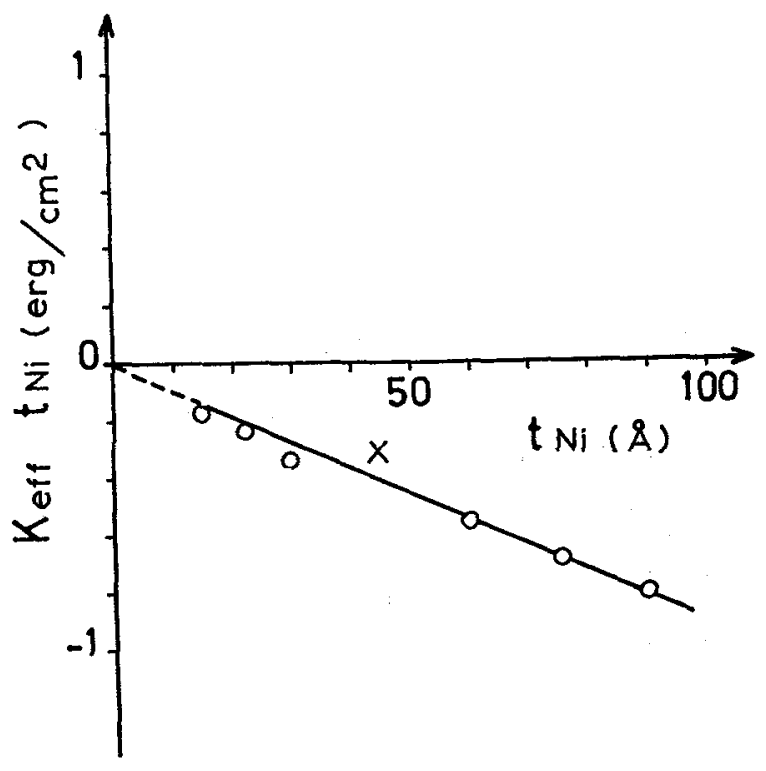

Fig. 1 The variation of the product of $\mathrm{K}_{\mathrm{eff}} \times \mathrm{t}(\mathrm{Ni})$ as a function of $\mathrm{t}(\mathrm{Ni})$ at $5 \mathrm{~K}$ 


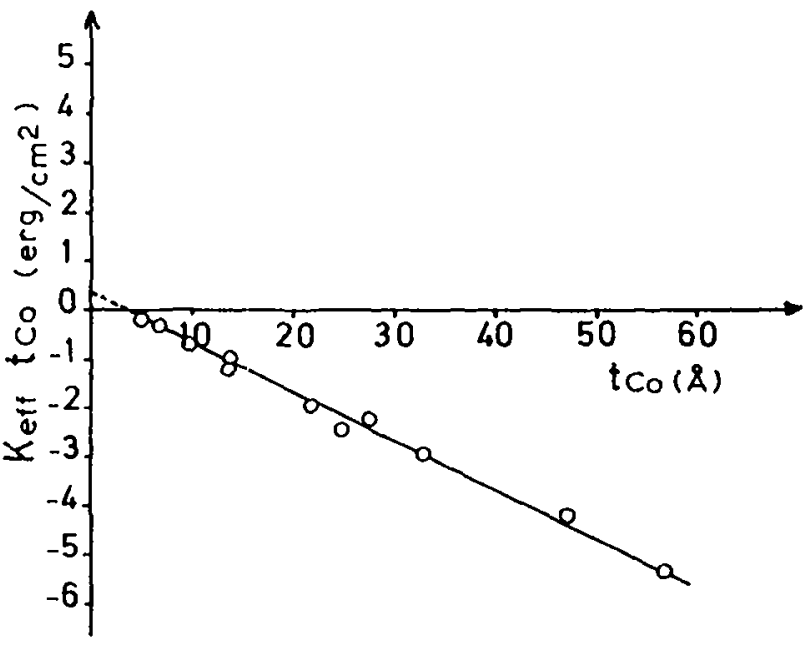

Fig. 3 The variation of the product of $\mathrm{Kefl}_{\mathrm{ef}} \mathrm{t}(\mathrm{CO})$ as a function of $\mathrm{t}(\mathrm{Co})$ at $5 \mathrm{~K}$

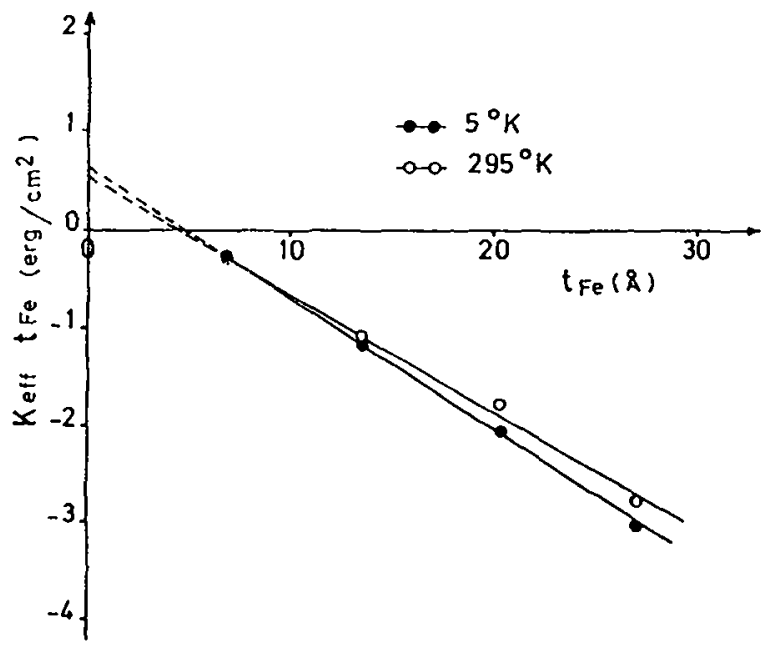

Fig. 2 The variation of the product of $K_{\text {eff }} \times t(F e)$ as a function of $t(F e)$ at $5 \mathrm{~K}$

This shows that as the Ni layer thickness is decreased, the magnetostriction has a tendency to change sign and become positive. Experiments with thinner Ni layers were not possible at room temperature, because of their lower Curie temperature.This has to be reckoned with when one tries to calculate the magnetoelastic energy contribution to the anisotropy in thin $\mathrm{Ni}$ layers. It is rather curious and not understood at present why surface Ni atoms do not contribute to the surface anisotropy although they do so to the magnetostriction.

The behaviour of the magnetostriction in Co/Ag multilayers is very different from what was observed for the anisotropy. Fig. 5 shows the complex dependence of the magneto-striction on the inverse Co layer thickness. For a thick Co layer the magnetostriction is found to be $-20 \mathrm{x}$ $10^{-6}$ which is smaller than that of hcp Co. The structure of Co in our case is expected to be fcc 


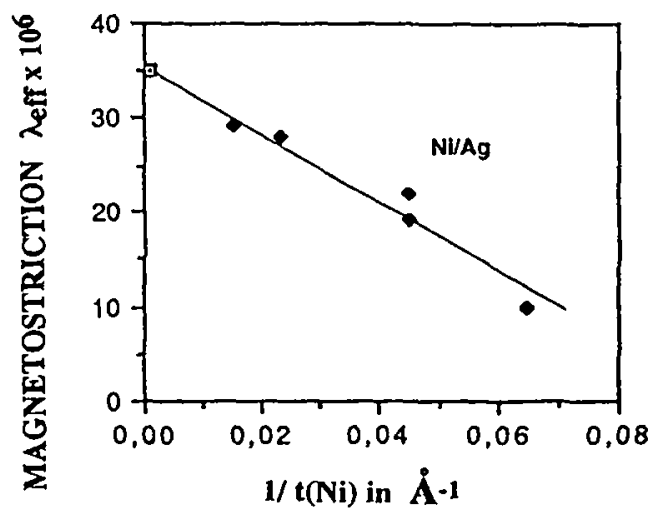

Fig. 4 The variation of $\lambda_{\mathrm{eff}}$ as a function of $\mathrm{t}(\mathrm{Ni})^{-1}$

rather than hexagonal because the resonance linewidths in these samples are rather low and on the order of 60 Oe. Furthermore the nuclear signal frequency of ${ }^{9} \mathrm{Co}$ was found to be centered at $222 \mathrm{MHz}$ which is slightly smaller than $228 \mathrm{MHz}$ for the h.c.p but closer to $217.5 \mathrm{MHz}$ for the fcc $\mathrm{Co}^{21}$. For $\mathrm{t}(\mathrm{Co})=60 \AA$ the magnetostriction changes sign and becomes positive and is almost independent of $t(\mathrm{Co})$ until one reaches $t(\mathrm{Co})=6 \mathrm{~A}$ when it is close to zero.

In order to explain this peculiar behaviour we had proposed that one should take into account the reduced symmetry when one approaches the nanostructured layers 22 . The main source of magnetostriction of cobalt based films are the dipolar and the single ion interactions. For both mechanisms the reduced symmetry leads to changes in the magnetostriction constant. Since the microscopic mechansim of magnetostriciton in such materials is not clarified yet, the contribution due to reduced symmetry has been modeled by the term :

$\lambda_{s}($ surface $)=\lambda_{s}{ }^{d}\left(a+c_{c o}\right)^{-1}$

Fitting procedure yielded the following parameters : $\lambda_{s}{ }^{d}=-350.10^{-6} \mathrm{~nm}, a=0.21 \mathrm{~nm}$, $\lambda_{s}($ surface $)=26.10^{-5} \mathrm{~nm}$ for $\lambda_{\mathrm{s}}$ (bulk) $=-28.10^{-6}$ (obtained for $1000 \AA$ thick film).

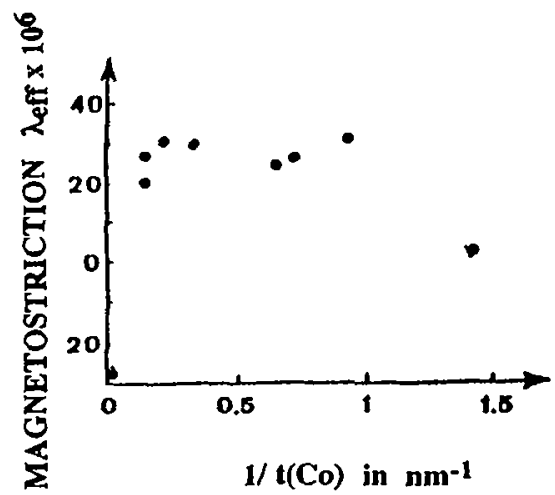

Fig. 5 The variation of $\lambda_{\text {eff }}$ as a function of $t(\mathrm{Co})^{-1}$ 
Let us now describe our results on $\mathrm{Co} / \mathrm{Pt}$ multilayers. This is a very interesting system as mentioned in the beginning, from both fundamental and practical points of view 9 . We grew these mutlilayers, either on glass or silicon kept either at $300 \mathrm{~K}$ or $475 \mathrm{~K}$. In some cases we also used a $100 \AA$ thick Pt buffer layer for the multilayer growth. We varied the Co layer thicknessin the range 4 to $30 \AA$ and kept Pt layer thickness fixed at 12 and $19 \AA$. The growth parameters will be designated as $(t(\mathrm{Co}), t(P t))^{N}$ where $t(\mathrm{Co})$ and $t(P t)$ indicate the layer thicknesses of $C o$ and $\mathrm{Pt}$ and the $\mathrm{N}$ indicates the number of Co layers. In all cases the top layer was Pt.

For thin layers of Co a strong uniaxial anisotropy is observed and the perpendicular $\mathrm{M}-\mathrm{H}$ loops are rectangular with coercivities on the order of $2 \mathrm{kOe}$ in agreement with published values. We have reported earlier on these multilayers $22-24$. We shall concentrate here mainly on the anisotropy and the magneto-optical studies.

The periodicity was verified by low angle $\mathrm{x}$-ray difraction and cross sectional transmission electron microscopy. The bi-layer thickness calculated from the low angle diffraction peaks agreed with that of the quartz monitor within $\pm 1 \AA$.

As the Co layer thickness decreases the magnetization expressed in terms of total volume of Co first decreases and then slightly increases. This is attributed to a contribution from the $\mathrm{Pt}$ atoms which get polarized by Co atoms 9 . The $\mathrm{M}-\mathrm{H}$ loops with the external field normal to the film plane are perfectly rectangular for the ML with $\mathrm{t}(\mathrm{Co})<10 \AA$. Fig. 6 a shows the perpendicular rectangular loop for the sample $A(4,12)^{15}$ deposited at $423 \mathrm{~K}$ on a $\mathrm{Pt}$ buffer layer $100 \AA$ thick. But for the sample $B(14,19)^{14}$, deposited under the same conditions as for $A$, the there is practically no remanance for the perpendicular loop as shown in Fig. $6 \mathbf{b}$.

(a)

(b)
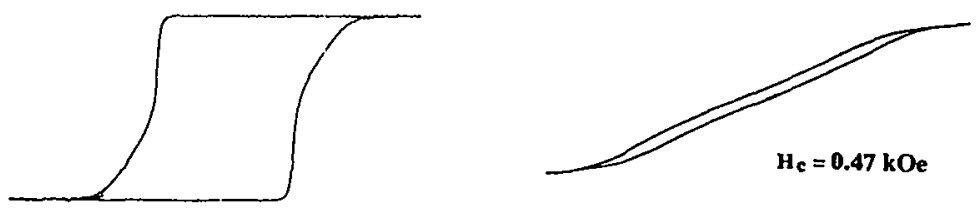

$\mathrm{H}_{\mathrm{e}}=2.0 \mathrm{kOe}$

Fig. 6 The M-H loops of Co/Pt multilayers, with (a) sample A and (b) sample B

Let us now discuss the results on the anisotropy. All the samples that are considered here have a fixed Pt layer thickness of 18-19 $\AA$. Fig. 7 shows the variation at $293 \mathrm{~K}$ of the product $\mathrm{K}_{\text {eff }} \times \mathrm{t}(\mathrm{Co})$ as a function of $\mathrm{t}(\mathrm{Co})$ for the samples deposited on glass substrates held at $300 \mathrm{~K}$ without $\mathrm{Pt}$ buffer layer. Some data points are also shown for samples deposited at 423 and 473 $\mathrm{K}$. It is seen that the growth temperatures influence strongly only when $\mathrm{t}(\mathrm{Co})<10 \AA$ and the $\mathrm{K}_{\mathrm{eff}}$ is found to be higher for ML deposited at higher temperature 22,23 . For the range $10 \AA<$ $t(\mathrm{Co})<30 \AA$ a linear dependence is obtained but for $t(\mathrm{Co})<10 \AA$, the data points show deviation. This is probably due to some modifications in the structure of the interface as the Co layer gets thinner which alters the site symmetry of the Co atom at the surface and therefore its contribution to the surface anisotropy. These results are similar to those generally reported in the literature 25 . It is seen that the cross-over to positive $K_{\text {eff }}$ occurs for $t(C o)=12 \AA$. A fit of the linear extrapolation gives $K_{s}=+0.6 \mathrm{erg} \cdot \mathrm{cm}^{-2}$. The results on silicon substrates were very similar although those on $\mathrm{MgO}(100)$ substrates are different and will be discussed below.

The Farady rotation (expressed per unit thickness of $\mathrm{Co}$ ) increases strongly as $\mathrm{t}(\mathrm{Co})$ is decreased as shown in Fig. 9. A model has been developed by Visnovsky to understand the magneto-optical effects in the multilayers which takes into consideration the boundary effects 26. This model was applied assuming the optical constants of bulk $C o$ and $P t$ and the $\Delta \mathrm{n}$ of Co as calculated from the published Faraday rotation of $\mathrm{Co}$. This calculation predicts an increase of only about $20 \%$ for the range of thickness considered here 27 . We therefore attribute our 


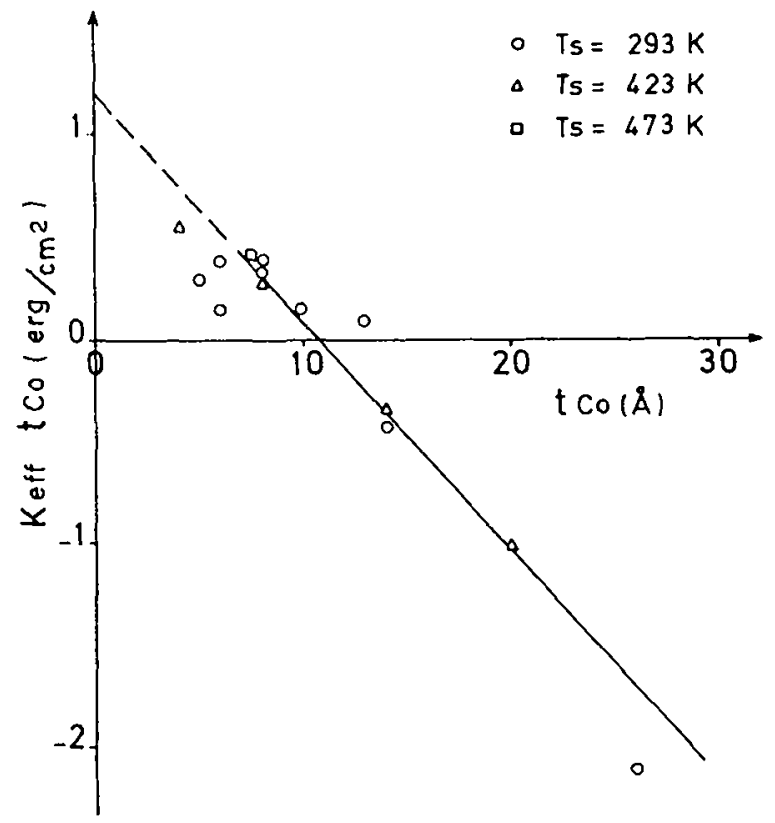

Fig. 7 The variation of the product of $K_{\operatorname{err}} x t(\mathrm{Co})$ as a function of $t(\mathrm{Co})$

result to the presence of an interfacial Co-Pt alloy which also contributes to the rotation. Assuming, as a first approximation, that the rotation of this alloy and bulk Co is the same, we find that the interface alloy is about 3 to $4 \AA$ thick. It is interesting to note that the magnetooptical studies also could serve to characterize the sample in certain cases.

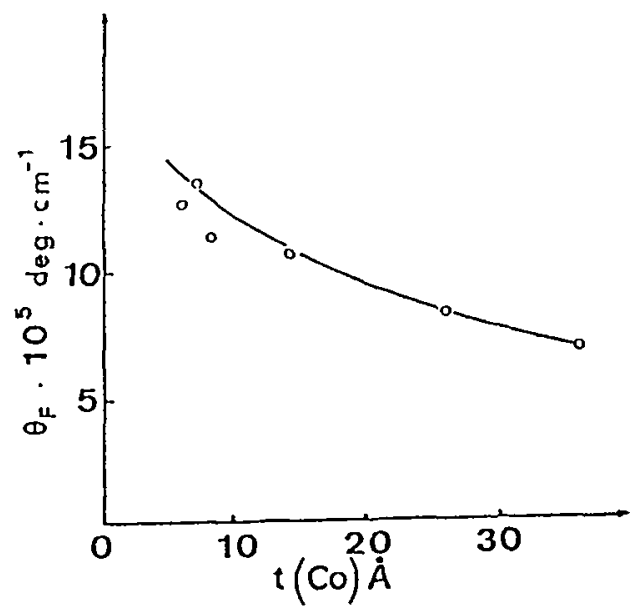

Fig. 8 The variation of the specific Faraday rotation as a function of $t(\mathrm{Co})$ 
The polar Kerr rotation for the sample $A$ is found to be -0.12 deg at $\lambda=6328 \AA$. But it increases to $-0.25 \mathrm{deg}$ near $\lambda=4000 \AA$ which is higher than what is found for the amorphous Tb-Fe alloy films.

We carried out NMR spin echo investigations in order to obtain some information on the interface material. The results are described only briefly here as we had reported on this very recently 28 . We studied at $2 \mathrm{~K}$ in zero field, three samples, $(26,32)^{6},(14,20)^{15}$ and $(10,26)^{16}$ deposited at $300 \mathrm{~K}$ and the results are shown in Fig. 9.

To identify the origin of the other contributions to the spectra, we prepared a Co3Pt alloy. The Co spin-echo spectrum of this is found to be centered near $208 \mathrm{MHz}$ which is rather high. This could arise from small lineshifts due to Pt nearest neighbours. The spectrum was broad which results from different $C$ hyperfine fields, depending upon the number of $P t$ nearest neighbours.

The observed CozPt spectrum fits reasonably well the low frequency tails (dotted lines) of the spectra of Co/Pt multilayers (Fig. 9). The proportion of Co atoms combined with Pt can be obtained by comparing the integrated signal intensity of $\mathrm{Co}_{3} \mathrm{Pt}$ with that from the sample (Fig. 9). The proportions are then calculated to be (accurate to within 30\%), 23, 55 and $90 \%$ for the samples with tCo $=26,14$ and $10 \AA$ respectively. This means that at each interface, a Co layer of about $4 \AA$ is alloyed. It is interesting to mention here that by studying the variation of the Faraday rotation with $\mathrm{t}(\mathrm{Co})$ one of us had evaluated the interface layer thickness to be about 3-4 $\AA 22$. So we have been able to characterize the interfacial alloy and it is believed that this material should play an important role in determining the uniaxial anisotropy.

It will be interesting to investigate the magnetostriction in $\mathrm{Co} / \mathrm{Pt}$ multilayers in order estimate the possible contribution from magnetoelastic energy and to compare its trend observed with that obtained in $\mathrm{Co} / \mathrm{Ag}$ system where the possiblitiy of alloying does not exist.

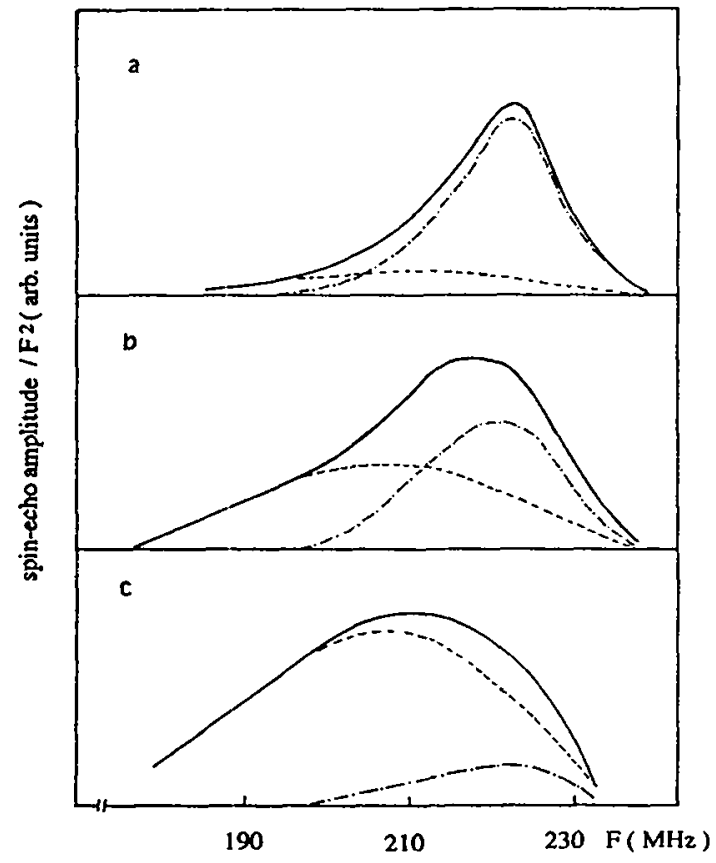

Fig. 9 Co spin echo spectrum at $2 \mathrm{~K}$ in Co/Pt multilayers a) $(26,32) 6$, b) $(14,20) 15$, and c) $(10,20) 16$. The dashed line represents the contribution from hcp Co and the dotted one from $\mathrm{CO}_{3} \mathrm{Pt}$ 
In conclusion the multilayers of fer rich possibilities of studying the anisotropy and magnetostriction in nanostructured materials where the classical approach is no longer valid. It is seen that the nature and the structure of the interface perturbs the properties and the magnetostriction seems to be more sensitive to these parameters. An atomic insight is needed for which more sophisticated tools of characterization are necessary. More experimental data on a well charcterized materials are welcome in order to refine the theoretical basis. Some of these multilayer systems such as $\mathrm{Co} / \mathrm{Pt}$ and $\mathrm{Co} / \mathrm{Pd}$ hold good promise for magneto-optical information storage of higher densities than achieved at present with amorphous rare earthtransition metal alloys. Further more these multilayers are chemically much more stable.

It is a pleasure to thank all my collaborators who contributed to this work. Part of this work was performed under the BRITE EURAM contract No. BREU 0153- C (TT) and is greatfully acknowledged.

\section{REFERENCES}

[1] See the various articles in "Growth, Characterization and properties of Ultra thin Magnetic films and Multi layers", edited by B.T.Jonker, J.P. Heremans and E.E. Marinero (Mater. Res. Soc. Symp. Proc. 151 Pittsburgh, (1989) ; Metallic Multilayers, edited by Chamberod A, and Hillairet, A, Materials Science Forum, 59 \& 60 (1990), Trans Tech Pub.Switzerland .

[2] Continenza, A, Massida, S, and Freeman, A.J, J. Magn, Magn. Mater. 78 1(1989) 95

[3] Elmers, H. J, Liu, G, and Gradmann, U, Phys. Rev. Lett, 63 (1989)566

[4] Krishnan R, and Tessier, M, J. App. Phys. 67 (1990) 5391

[5] L. Néel, L, C.R. Acad. Sci., 237 (1953) 1468

[6] Gradmann, U, J. Magn. Magn. Mater., $54-57$ (1986) 733

[7] Gay J.G, and Roy Richter, Phys. Rev. Lett. 56 (1986) 2728

[8] Heinrich, B, Urquhart, K.B, Arrot, A.S, Cochran, J.F, Myrtle K, and Purcell, S.T, Phys. Rev. Lett. 59 (1987) 1756

[9] Carcia, P.F, Meinhaldt, A.D, and Suna, A, Appl. Phys. Lett., 47 (1985) 178 ; den Broeder, F.J.A, Donkersloot, H, Draaisma, H.J.G, and de Jonge, W.J.M, J. Appl. Phys., 61 (1985) 4137

[10] Krishnan, R, Cagan, V, Porte, M, and Tessier, M, J. Magn. Magn. Mater. 78 (1989) 122

[11] de Lacheisserie, E, Proc. 5 th Int. Conf. Physics of Magnetic Material, Poland, ed. W. Gorzkowski, M. Gutowski, H.K. Lachowich and H. Szymczak, World Scientific, Singapore, Vew Jersey, London, Honkong, 1990 pl64.

[12] Zuberek, R, Szymczak, H, Krishnan, R, Youn, K.B, and Sella, C, IEEE Trans. Mag. MAG 23 (1987) 3699

[13] Baibich, M.N, Broto, J.M, Fert, A, Nguyen Van Dau, Petroff, F, Etiene, P, Creuzet, G, Friedrich, A, and Chazelas, J, Phys. Rev. Lett., 61 (1988) 2472

[14] Parkin, S.S, Bhadra, R, and Roche, K.P, Phys. Rev. Lett. 66 (1991) 2152

[15] Krishnan, R, J. Magn. Magn. Mater. 50 1(1985) 89

[16] Wosik, J, Nesteruk, Zbieronowski, W, Sienkiewicz, A, J. Phys. E : Sci. Instrum. 11 (1988) 200

[17] Chun Li, Freeman, A.J, and Fu, C.L, J. Magn. Magn. Mater. 83 (1989) 51

[18] Krishnan, R, J. Magn. Magn. Mat. 103 (1991) 47

[19] den Broeder, F.J.A, Hoving, W, and Bloemen, P.J.H, J. Magn. Mag. Mat. 93 (1991) 562

[20] Zuberek, R, Szymczak, H, Krishnan, R, and Tessier, M, J. de Phys. suppl. 49 (1988) C81761

[21] Le Dang , K, Private communication

[22] Krishnan, R, Porte M, and Tessier, M, IEEE Trans. Mag. 262727 (1990)

[23] Krishnan, R, Porte, M, and Tessier, M, J. Magn. Soc. Jap. 15 S1 21 (1991)

[24] Krishnan, R, Lassri, H, Porte, M, Tessier, M, and Flevaris, N.K, Mat. Res. Symp. Proc.

232 (1991) 91

[25] Hashimoto, S, Ochiai, Y, and Aso, K, J. Appl. Phys., 67 (1990) 2136

[26] Visnovsky, S, J. Magn. Soc. Jap. 15 S 1 (1991)

[27] Visnovsky, $S$, Private communication

[28] Le Dang, K, Veillet, P, Krishnan, R and Lassri, H, to appear in Appl. Phys. Lett. (1992) 